\title{
Ensemble pédagogique expérimental hacheur onduleur
}

\author{
Jean-Jacques HUSELSTEIN, Thierry MARTIRÉ \\ Laboratoire d'Électrotechnique de Montpellier (LEM) - Université Montpellier II, \\ CC079, Place Eugène Bataillon, 34095 MONTPELLIER CEDEX 5, \\ huselstein@univ-montp2.fr, martire@univ-montp2.fr
}

\begin{abstract}
RESUME Cet article présente un dispositif expérimental « hacheur-onduleur » que nous utilisons pour une grande partie de nos travaux pratiques d'électronique de puissance. Il est composé d'un étage de puissance (onduleur de tension triphasé à IGBT) et d'une carte de commande basée sur un FPGA. Selon la configuration choisie par l'utilisateur, cet ensemble peut constituer un convertisseur parmis une liste préétablie allant du simple hacheur série non réversible jusqu'à l'onduleur triphasé à modulation de largeur d'impulsion avec une loi de commande de type U/f = constante. La constitution du système expérimental ainsi que nombreux exemples d'utilisation en travaux pratiques illustrés par des relevés expérimentaux sont présentés dans cet article.
\end{abstract}

Mots clés : Onduleur, hacheur, MLI, IGBT, commande, puissance, modulation.

\section{INTRODUCTION}

\subsection{Nos motivations pour réaliser ce dispositif}

Notre motivation première était de réaliser un dispositif de commande numérique pour deux onduleurs pédagogiques SEMIKRON dont nous disposions (ces derniers sont dépourvus de commande et donc inutilisables seuls). Partant de là, tant qu'à concevoir un circuit de commande numérique pour un onduleur triphasé, pourquoi ne pas aussi étendre ses possibilités à d'autres fonctions de conversion? Ensuite nous souhaitions pouvoir faire travailler tout un groupe d'étudiants simultanément sur le même sujet. Il est alors indispensable de disposer d'autant de cartes de commande et de circuits de puissance que de tables de TP. La carte de commande a donc été réalisée en dix exemplaires. L'onduleur SEMIKRON (partie puissance) étant trop coûteux pour en acquérir dix unités, nous avons conçu et dupliqué en dix exemplaires un circuit de puissance d'onduleur triphasé économique et performant basé sur des IGBT 600V-12A (avec diode intégrée) et un circuit de commande rapprochée à décalage de niveau IR2136.

\subsection{Constitution}

Cet ensemble « hacheur-onduleur » est donc constitué d'une carte de commande totalement numérique basée sur un FPGA et d'une partie puissance qui est un onduleur de tension triphasé. Quelque soit le mode d'utilisation, le câblage interne de la structure de puissance reste inchangé. C'est seulement la commande des transistors de puissance qui détermine la topologie et la fonction de conversion obtenue. Le mode de fonctionnement est choisi sur la carte de commande ainsi que tous les paramètres de fonctionnement (fréquence de découpage, rapport cyclique, fréquence de modulation, profondeur de modulation). Ces réglages sont effectués par des codeurs incrémentaux qui donnent à l'utilisateur l'impression d'agir sur des potentiomètres. Ces réglages se font obligatoirement avec l'oscilloscope : ceci permet une simplicité dans la conception du système (pas d'afficheurs) mais a aussi des avantages pédagogiques. Les étudiants doivent savoir utiliser correctement et efficacement un oscilloscope et cela évite le côté « TP presse bouton ».

\subsection{Utilisation en salle de TP}

Cet ensemble «commande et puissance » est utilisé dans nos TP depuis octobre 2003. Dix ensembles sont en fonction, un par table. Ils sont utilisés en licence Ingénierie Électrique, en licence L1 et L2 STPI EEA, en Master STPI EEA, en $1^{\text {ère }}$ année école d'ingénieur Polytech MEA.

Ce dispositif nous permet de faire aussi bien ou mieux les TP que nous faisions déjà auparavant: hacheur série et onduleur triphasé sur onduleur industriel. Remarque : un onduleur industriel est très bien pour alimenter un moteur, mais il est d'une utilisation difficile pour la pédagogie de l'électronique de puissance en raison des nombreuses protections et de ses modes de MLI et de commande vectorielles complexes. Le fonctionnement avec une charge autre qu'un moteur à courant alternatif est souvent impossible.

Ce nouveau dispositif nous permet aussi et surtout de faire de nouveaux TP d'électronique de puissance avec différents modes de commande qui n'étaient pas possibles avant, la commande adéquate faisant défaut.

\section{PARTIE PUISSANCE}

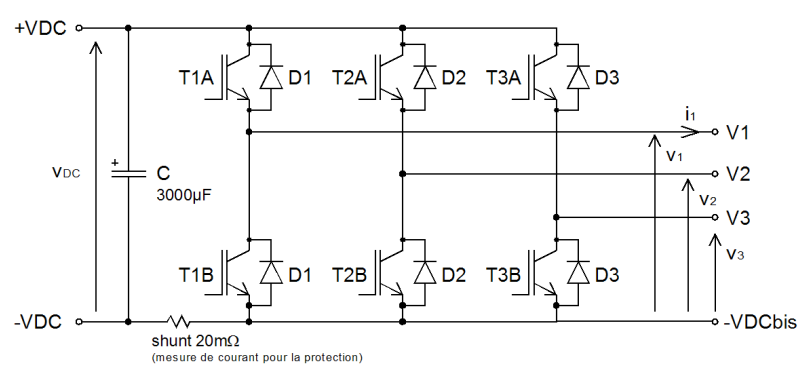

Fig 1 : Structure de la carte de puissance.

La partie puissance est un onduleur de tension triphasé constitué de 6 IGBT avec diode intégrée en boîtier TO247 et de deux condensateurs électrolytiques $330 \mu \mathrm{F}-450 \mathrm{~V}$ montés en parallèle. Les IGBT sont pris 
« en sandwich » entre le circuit imprimé et le radiateur pour assurer une construction compacte avec une inductance parasite minimale. Les commandes de grilles sont assurées par un circuit intégré IR2136 qui gère les décalages de niveau pour les IGBT supérieurs. Cette carte comprend aussi une protection en courant et une protection contre les surtensions du bus continu. La protection en courant est assurée par une résistance shunt CMS à très faible inductance parasite et deux comparateurs rapides LM319. Le seuil de protection est fixé à $10 \mathrm{~A}$. Les sorties logiques des comparateurs retournent à la carte de commande qui assure la gestion des défauts. Le temps de réaction total de la protection (détection + blocages IGBT) est suffisamment court (environ 600ns) pour assurer une protection des IGBT même dans le cas d'un court-circuit franc en sortie. Une faible inductance $(22 \mu \mathrm{H})$ placée en série sur chacune des phases de sortie limite la vitesse de croissance du courant pendant ces défauts. Avec une tension d'alimentation continue de $400 \mathrm{~V}$ le dépassement du seuil de protection en courant est limité à environ $12 \mathrm{~A}$.

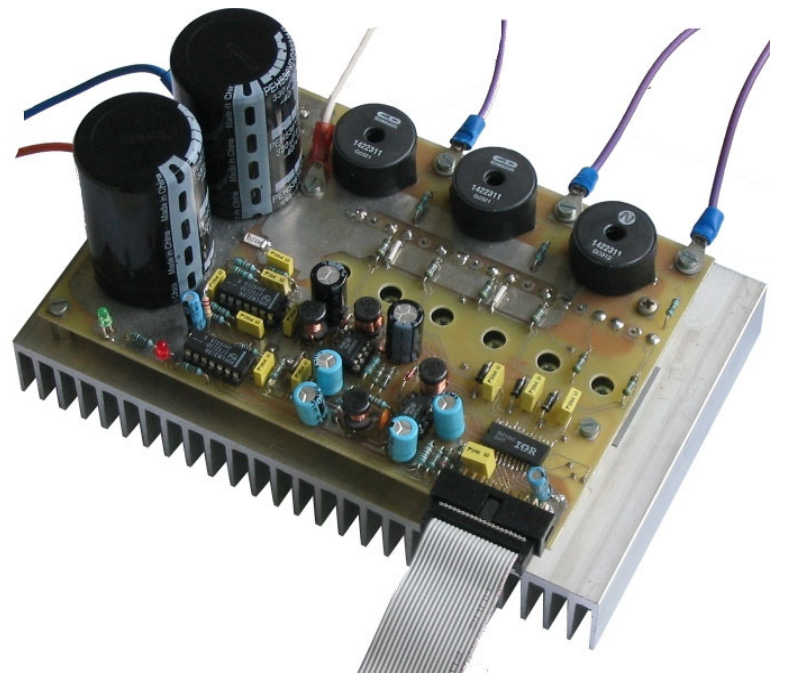

Fig 2: Vue de la carte de puissance extraite de son boitier.

\section{PARTIE COMMANDE}

Cette carte de commande est totalement numérique. Elle est basée sur un FPGA (circuit logique programmable) qui intègre l'intégralité des fonctions de commande. La liaison avec la carte de puissance se fait par un câble en nappe sur lequel transitent les signaux de commandes des six IGBT et les trois signaux de défaut (surintensité positive, surintensité négative et surtension du bus continu) renvoyés par la partie puissance. La carte de commande comporte deux boutons poussoirs permettant de sélectionner le mode de commande. Les paramètres de fonctionnement (fréquence de découpage, rapports cyclique, fréquence et amplitude de modulation en mode onduleur,) sont à régler par de boutons rotatifs reliés à des codeurs incrémentaux. Le FPGA utilisé est un circuit Altera FLEX10K10 associé à sa mémoire de configuration EPC2 [1]. Cette mémoire de configuration peut être reprogrammée pour les évolutions éventuelles. Le FPGA être directement configuré depuis un PC pendant les phases de mise au point. Le développement du projet a été réalisé avec le logiciel Altera Max+Plus II web edition (gratuit) actuellement remplacé par Quartus II web edition (également gratuit) [1].

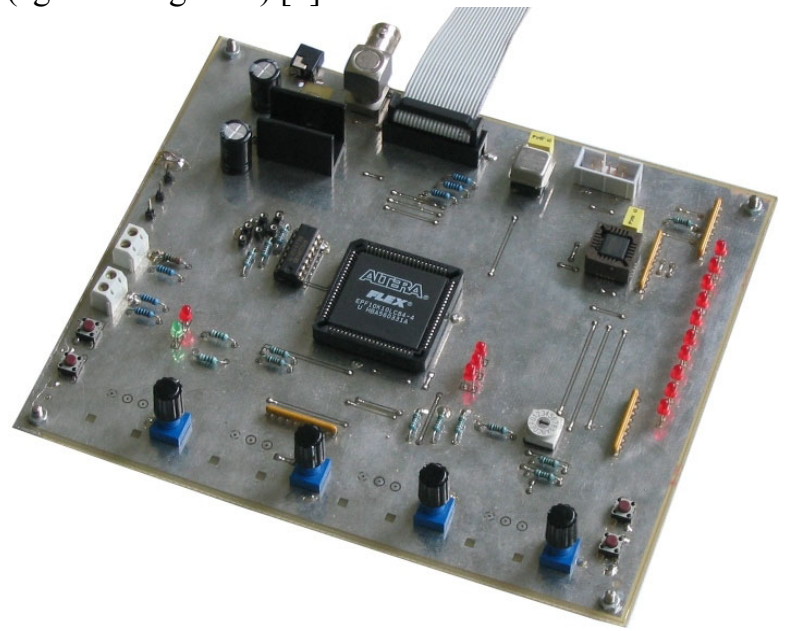

Fig 3 : Vue de la carte de commande.

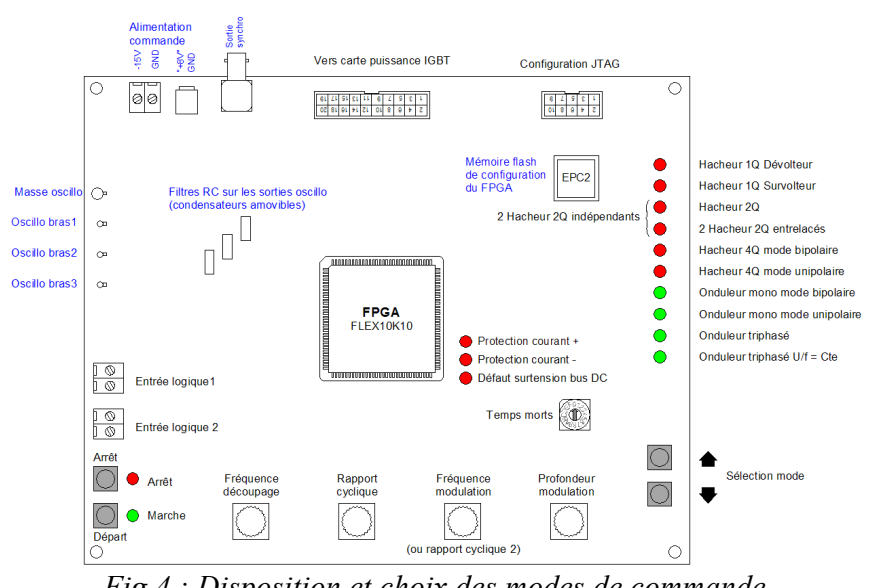

3.1 Modulation de largeurs d'impulsions

La MLI est de type intersective. Le signal triangulaire de référence (codé sur 8 bit) à la fréquence de découpage est obtenu par une synthèse directe de fréquence par intégration numérique de la consigne de fréquence.

\subsection{Modulation sinusoïdale}

La référence sinusoïdale est obtenue également par une synthèse numérique directe de la fréquence de modulation. Cette synthèse directe fournit un signal angulaire (codé sur 8 bit) appliqué à une table sinus. Ensuite une opération de multiplication (8bit $\mathrm{x} 8$ bit avec un résultat tronqué aux 8bits de poids fort) permet de régler l'amplitude de modulation.

\subsection{Réglages par codeurs incrémentaux}

En association avec une machine d'états et des fonctions de comptage/décomptage intégrées dans le FPGA ces codeurs se comportent un peu comme des potentiomètres. Ils permettent de régler de manière quasi 
continue sur une large plage (tableau 1) les paramètres fréquence de découpage, rapport cyclique (fonctionnements hacheurs), fréquence de modulation et profondeur de modulation (fonctionnements onduleurs).

\begin{tabular}{|l|l|l|}
\hline $\begin{array}{l}\text { Fréquence de } \\
\text { découpage }\end{array}$ & $610 \mathrm{~Hz}$ à $38,76 \mathrm{kHz}$ & pas de $305 \mathrm{~Hz}$ \\
\hline $\begin{array}{l}\text { Rapport cyclique en } \\
\text { mode hacheur }\end{array}$ & 0 à $99,65 \%$ & pas de $0,387 \%$ \\
\hline $\begin{array}{l}\text { Fréquence de } \\
\text { modulation }\end{array}$ & $0,3125 \mathrm{~Hz}$ à $101,3 \mathrm{~Hz}$ & pas de $0,395 \mathrm{~Hz}$ \\
\hline $\begin{array}{l}\text { Amplitude de } \\
\text { modulation }\end{array}$ & 0 à $98,5 \%$ & pas de $1,56 \%$ \\
\hline $\begin{array}{l}\text { Modulation en mode } \\
\text { U/f }=\text { constante }\end{array}$ & $\begin{array}{l}\text { proportionnelle à } \mathrm{F} \text { B BF de } 0 \text { à } 25,03 \mathrm{~Hz} \text { puis } \\
\text { constante }(98,5 \%) \text { jusqu'à } 101,3 \mathrm{~Hz}\end{array}$ \\
\hline
\end{tabular}

Tableau 1 : Plages de réglage des paramètres de commande.

Les codeurs incrémentaux utilisés intègrent deux interrupteurs mécaniques délivrant 32 impulsions par tour. Les 2 sorties sont déphasées d'un quart de période ce qui permet de déterminer le sens de rotation (fonctionnement classique). Nous avons choisi de compter les fronts montant et descendant de la voie A, l'état de la voie B lors de ces fronts définissant le sens de rotation. La figure 5 représente les diagrammes décrivant le fonctionnement des machines d'états assurant la gestion de ces codeurs ainsi que deux exemples de fonctionnement. Les machines d'états et les compteurs correspondants (un ensemble par codeur) sont implantés en langage AHDL dans le projet global de la carte de commande.

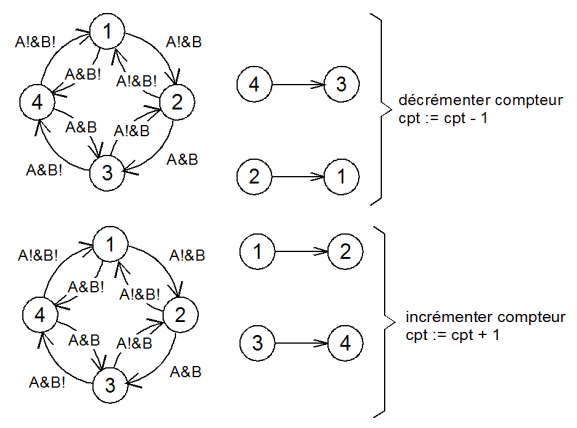

Fig 5 : Diagrammes d'états pour la gestion des codeurs.

\section{EXEMPLES D'UTILISATIONS}

\subsection{Contextes des essais présentés}

La plupart des relevés présentés ici ont été effectués avec une tension d'alimentation continue égale à $60 \mathrm{~V}$ issue d'une alimentation de laboratoire. C'est généralement à partir de ce type de source que nous faisons travailler nos étudiants pour des raisons de simplicité et surtout de sécurité des personnes et des équipements. Dans le cadre de projets plus avancés nous utilisons aussi des alimentations continue isolées 0 à $270 \mathrm{~V}$ disponibles sur certaines de nos tables de manipulation ce qui nous permet de travailler avec des puissances plus importantes (alimentation machine asynchrone, injection de puissance sur le réseau, onduleur à résonance $2 \mathrm{~kW}$, etc.).
Les résultats présentés ici ont été enregistrés sur des oscilloscopes numériques deux voies. Lorsque plus de deux voies sont présentées simultanément il s'agit d'un montage de copies d'écran. La correspondance temporelle a pu être facilement assurée grâce à la sortie de synchronisation présente sur la carte de commande.

\subsection{Hacheur série}

Dans ce mode seul le transistor $\mathrm{T}_{1 \mathrm{~A}}$ est commandé avec un rapport cyclique fixe $\alpha$ (réglé par le $2^{\text {ème }}$ codeur) et une fréquence de découpage $F_{\text {déc }}$ (réglée par le $1^{\text {er }}$ codeur). Les autres transistors sont toujours maintenus à l'état bloqué. La fonction de diode de roue libre est assurée par la diode $\mathrm{D}_{1 \mathrm{~B}}$.

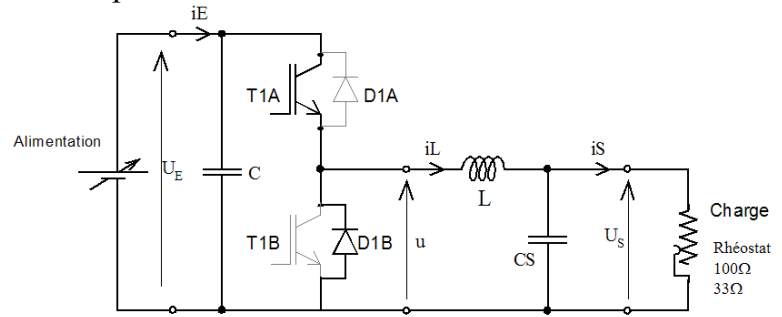

Fig 6 : Utilisation en mode " hacheur série ».

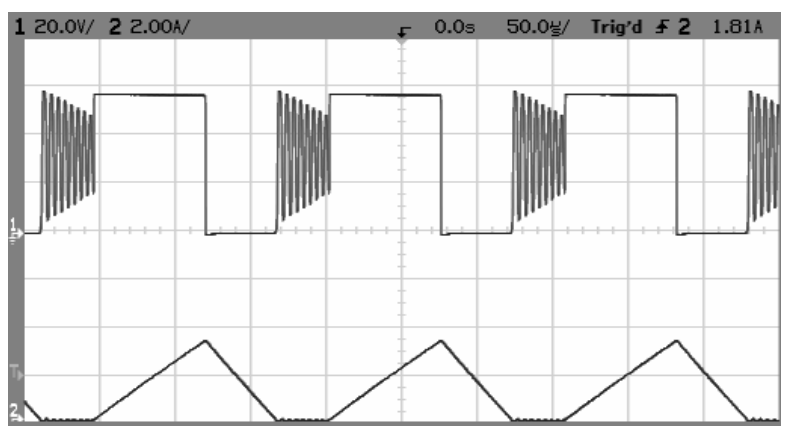

Fig 7 : Hacheur 1 quadrant en conduction discontinue. Haut : tension de sortie $u$. Bas : courants de sortie $i_{L}$.

\subsection{Hacheur parallèle}

Dans ce mode, seul le transistor $\mathrm{T}_{1 \mathrm{~B}}$ est commandé avec un rapport cyclique $\alpha$ et une fréquence de découpage $F_{\text {déc }}$. Tous les autres transistors sont bloqués.

Les entrées et sortie du convertisseur sont inversées par rapport à un hacheur série: la source de tension d'alimentation du hacheur est connectée en série avec l'inductance de lissage L, la charge est connectée en parallèle avec le condensateur de découplage C.

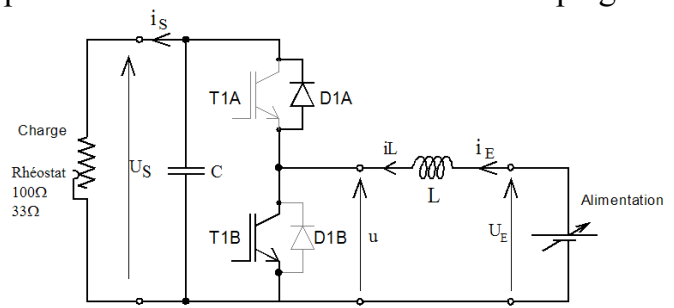

Fig 8: Utilisation en mode " hacheur parallèle ».

\subsection{Hacheur 2 quadrants}

Les transistors $T_{1 \mathrm{~A}}$ et $\mathrm{T}_{1 \mathrm{~B}}$ sont commandés de manière complémentaire avec un rapport cyclique $\alpha$ et une 
fréquence de découpage $F_{\text {déc }}$. Les autres transistors sont toujours bloqués.

\subsection{Deux hacheur 2 quadrants entrelacés}

Les transistors $\mathrm{T}_{1 \mathrm{~A}}$ et $\mathrm{T}_{1 \mathrm{~B}}$ sont commandés avec un rapport cyclique $\alpha$ et une fréquence de découpage $F_{\text {déc }}$. Il en est de même pour les transistors $T_{2 A}$ et $T_{2 B} d u$ deuxième bras mais avec un décalage temporel d'une demie période. Le filtre de sortie est composé de deux inductance de lissage et un condensateur. Un des intérêt de cette structure bien connue est de diviser par quatre l'amplitude maximale de l'ondulation de tension de sortie pour une même fréquence de découpage et pour un même volume total d'éléments réactifs. L'entrelacement est utilisé depuis longtemps dans les entraînements de forte puissance avec moteur à courant continu (traction ferroviaire par exemple) et plus récemment, avec un nombre de " phases » plus élevé, dans les convertisseurs continu-continu non isolés de très faible tension et fort courant utilisés dans les systèmes microélectroniques (ex : $3,3 \mathrm{~V}$ vers $1,2 \mathrm{~V}$ 80A pour un processeur de type Pentium).

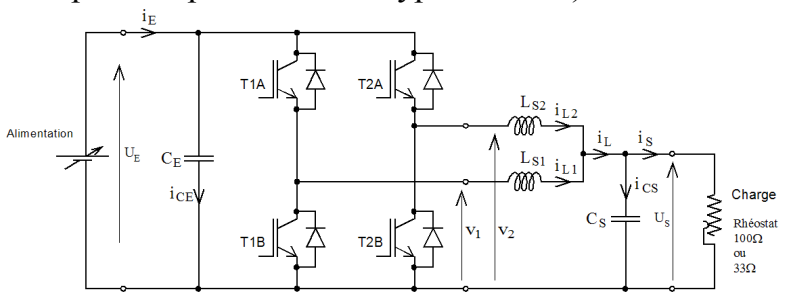

Fig 9: Mode "deux hacheurs 2 quadrants entrelacés».

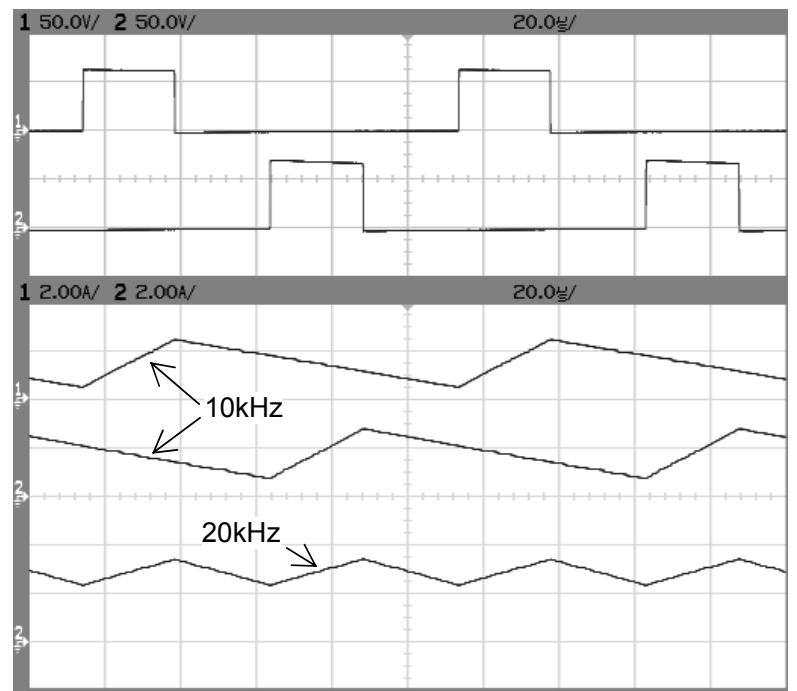

Fig 10 : Deux hacheurs 2 quadrants entrelacés.

Haut : tensions de sortie $v_{1}$ et $v_{2}$. Bas : courants $i_{L 1}$, $i_{L 2}$ et $i_{L}$.

\subsection{Deux hacheurs 2 quadrants en opposition}

Ce mode de commande permet de faire fonctionner deux hacheurs et deux machines à courant continu à aimants permanents en opposition. Chacune de machines peut fonctionner soit en moteur soit en génératrice. Le courant de sortie de chacun des hacheurs peut être positif ou négatif. La méthode d'opposition évite les difficultés dues à la non réversibilité des alimentations de laboratoire. L'alimentation ne fournit au système que les pertes (des hacheurs et des moteurs). La puissance convertie peut être beaucoup plus élevée que la puissance d'alimentation [2].

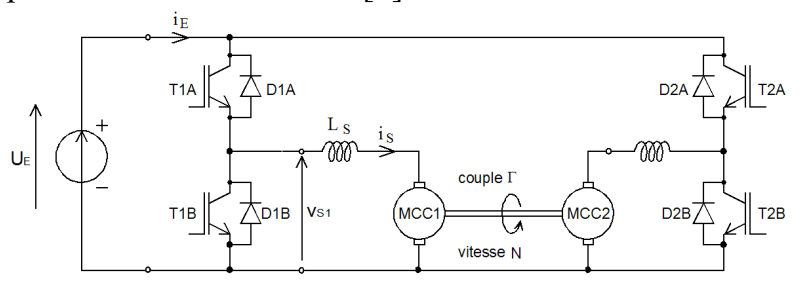

Fig 11 : Fonctionnement en opposition de deux machines à courant continu avec deux hacheurs 2 quadrants.

Les transistors $\mathrm{T}_{1 \mathrm{~A}}$ et $\mathrm{T}_{1 \mathrm{~B}}$ sont commandés avec un rapport cyclique $\alpha_{1}$ et une fréquence de découpage

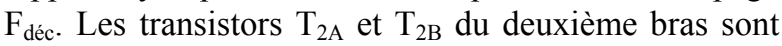
commandés à la même fréquence de découpage avec un rapport cyclique $\alpha_{2}$ (réglé par le $3^{\text {ème }}$ codeur).

Les arbres des deux machines à courant continu sont solidaires. Si les deux machines ont les mêmes caractéristiques (et surtout une même constante électromécanique) leurs tensions d'alimentation sont très semblables. Il doit donc en être de même pour les rapports cycliques des deux hacheurs. Le courant moyen $\mathrm{I}_{\mathrm{S}}$ circulant dans les machines est réglé par la légère différence appliquée entre ces deux rapports cycliques, ce courant pouvant être positif ou négatif.

$$
I_{S}=\left(\alpha_{1}-\alpha_{2}\right) \frac{U_{E}}{2 R} \quad \begin{aligned}
& \begin{array}{l}
\text { si les chutes de tension dans } \\
\text { le hacheur sont négligées }) \\
R \text { : résistance interne des MCC }
\end{array}
\end{aligned}
$$

En pratique nous demandons aux étudiants de régler la vitesse de rotation du banc par un réglage du rapport cyclique $\alpha_{1}$ avec seulement la machine MCC1 connectée. Puis d'obtenir la même vitesse de rotation en ajustant le rapport cyclique $\alpha_{2}$ avec seulement la machine MCC2 connectée. Ensuite, les deux machines étant connectées, le courant (positif ou négatif) est réglé par des légères variations du rapport cyclique $\alpha_{2}$.

\subsection{Hacheur 4 quadrants - commande bipolaire}

Les transistors $\mathrm{T}_{1 \mathrm{~A}}$ et $\mathrm{T}_{1 \mathrm{~B}}$ du $1^{\mathrm{er}}$ bras sont commandés avec un rapport cyclique $\alpha$ et une fréquence de découpage $F_{\text {déc }}$. Les transistors $T_{2 A}$ et $T_{2 B}$ du deuxième bras sont commandés avec un rapport cyclique $\alpha_{2}=1-\alpha$ obtenu par une simple inversion logique des commandes de $T_{1 \mathrm{~A}}$ et $\mathrm{T}_{1 \mathrm{~B}}$.

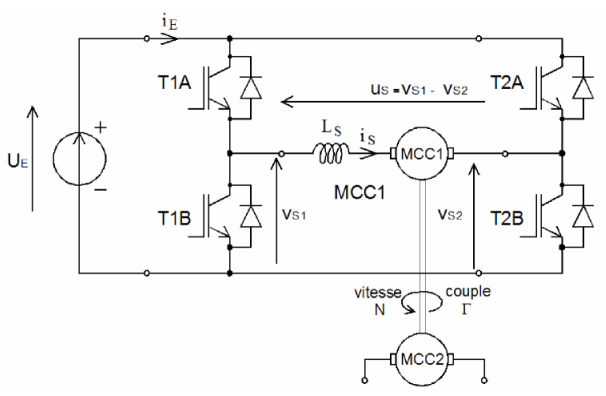

Fig 12 : Hacheur 4 quadrants. 


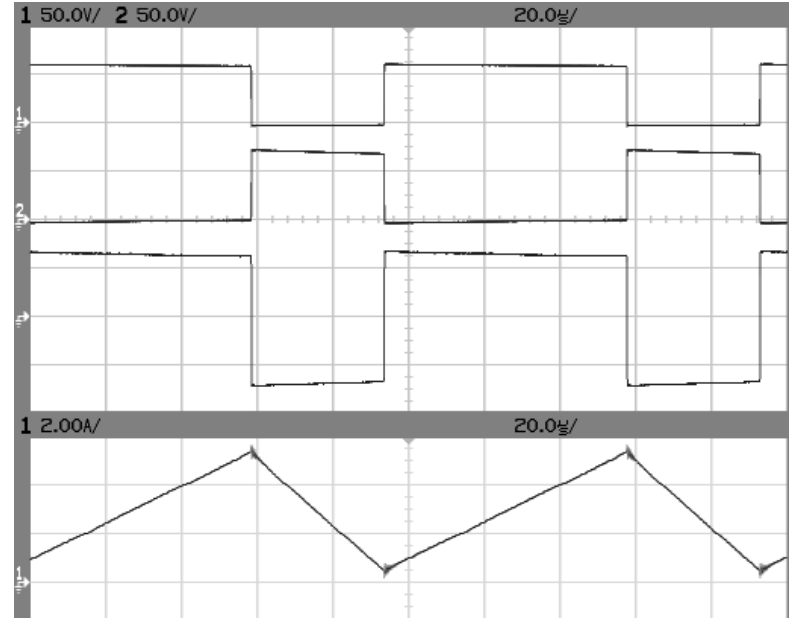

Fig 13 : Hacheur 4 quadrants en commande bipolaire. Haut : tensions de sortie $v_{S 1}, v_{S 2}$ et $v_{S}$. Bas : courant $i_{L}$.

\subsection{Hacheur 4 quadrants - commande unipolaire}

Les transistors $\mathrm{T}_{1 \mathrm{~A}}$ et $\mathrm{T}_{1 \mathrm{~B}}$ sont commandés avec un rapport cyclique $\alpha$ et une fréquence de découpage $F_{\text {déc }}$. Les transistors $T_{2 A}$ et $T_{2 B}$ du deuxième bras sont commandés avec un rapport cyclique $\alpha_{2}=1-\alpha$ obtenu à partir d'une deuxième modulation de largeur d'impulsion avec un décalage temporel d'une demie période. Ce mode de commande bien connu permet de réduire très fortement l'ondulation de courant sans modifier la fréquence de découpage.

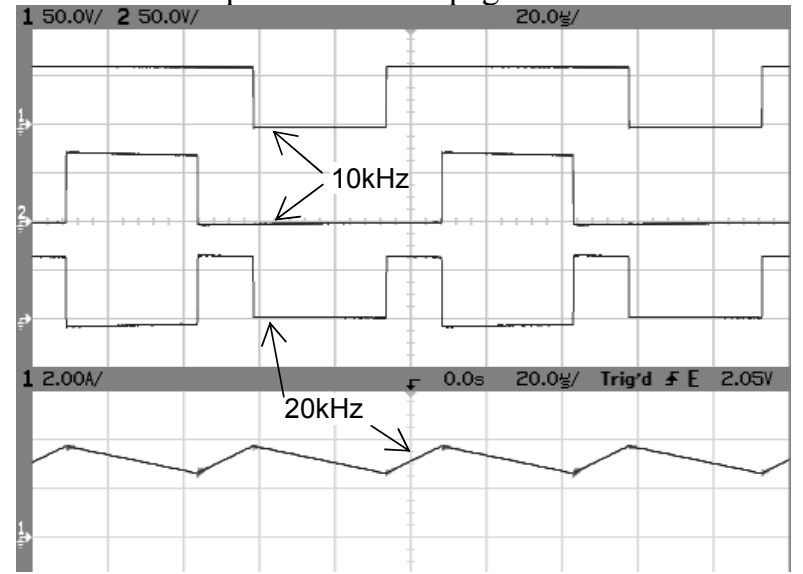

Fig 14 : Hacheur 4 quadrants en commande unipolaire. Haut : tensions de sortie $v_{S 1}, v_{S 2}$ et $v_{S}$. Bas : courant $i_{L}$.

\subsection{Onduleur monophasé - commande bipolaire}

Les transistors $\mathrm{T}_{1 \mathrm{~A}}$ et $\mathrm{T}_{1 \mathrm{~B}}$ sont commandés comme dans le mode hacheur 4 quadrants, mais le rapport cyclique fixe $\alpha$ est remplacé par un rapport cyclique variable en fonction du temps selon une loi sinusoïdale avec une fréquence de modulation $\mathrm{F}_{\mathrm{BF}}$ (réglée par le $3^{\text {ème }}$ codeur) avec $\alpha=0,5+\mathrm{P}_{\mathrm{MOD}} \sin 2 \pi \mathrm{F}_{\mathrm{BF}}$. Les transistors $\mathrm{T}_{2 \mathrm{~A}}$ et $\mathrm{T}_{2 \mathrm{~B}} \mathrm{du}$ deuxième bras sont commandés avec un rapport cyclique $\alpha_{2}=1-\alpha$ obtenu par une simple inversion logique des commandes de $\mathrm{T}_{1 \mathrm{~A}}$ et $\mathrm{T}_{1 \mathrm{~B}}$.

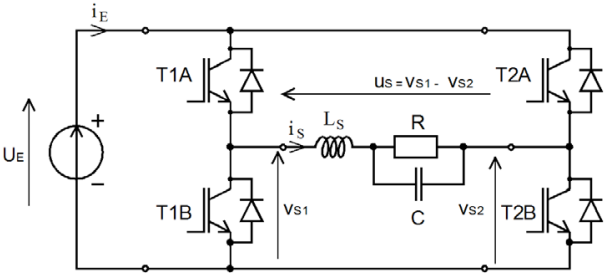

Fig 15 : Onduleur monophasé (ou hacheur 4 quadrants) sur une charge résistive avec un filtre $L C$.

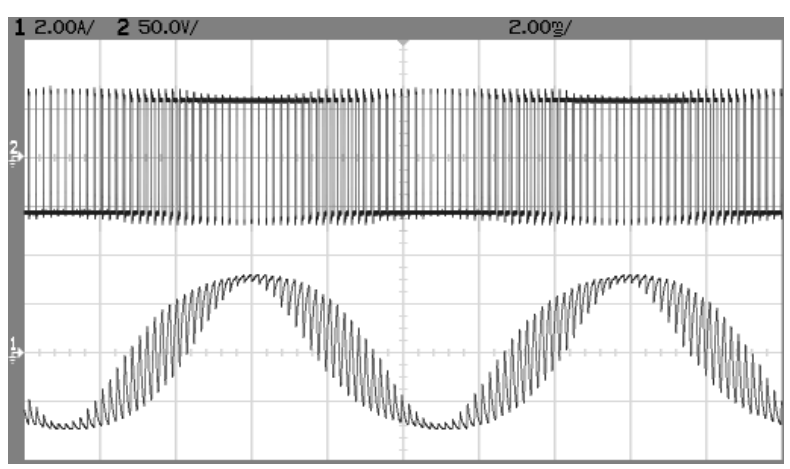

Fig 16: Onduleur monophasé en commande bipolaire. Haut : tension de sortie $v_{S}$. Bas : courant $i_{S}$.

4.10 Onduleur monophasé - commande unipolaire Les transistors $T_{1 \mathrm{~A}}$ et $\mathrm{T}_{1 \mathrm{~B}}$ sont commandés comme dans le mode hacheur 4 quadrants, mais le rapport cyclique fixe est également remplacé par un rapport cyclique variable en fonction du temps selon une loi sinusoïdale avec $\alpha=0,5+\mathrm{P}_{\mathrm{MOD}} \sin 2 \pi \mathrm{F}_{\mathrm{BF}}$. Les transistors $\mathrm{T}_{2 \mathrm{~A}}$ et $\mathrm{T}_{2 \mathrm{~B}}$ du deuxième bras sont commandés avec un rapport cyclique $\alpha_{2}=1-\alpha$ obtenu à partir d'une deuxième modulation de largeur d'impulsion avec un décalage temporel d'une demie période. Comme pour le hacheur 4 quadrants, l'intérêt de cette commande réside dans la forte réduction de l'ondulation de courant.

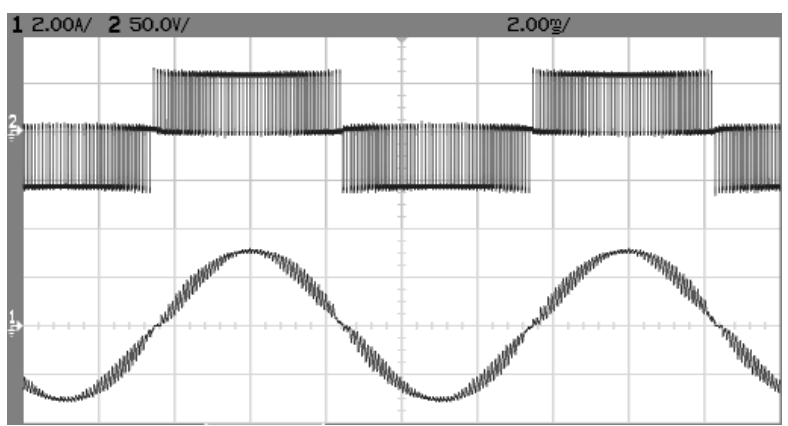

Fig 17 : Onduleur monophasé en commande unipolaire. Haut : tension de sortie $v_{S}$. Bas : courant $i_{S}$.

\subsection{Onduleur triphasé}

Comme pour les modes onduleur monophasé, les transistors $T_{1 \mathrm{~A}}$ et $\mathrm{T}_{1 \mathrm{~B}}$ du premier bras sont commandés avec un rapport cyclique variable en fonction du temps selon une loi sinusoïdale avec une fréquence de modulation $\mathrm{F}_{\mathrm{BF}}$ avec $\alpha_{1}=0,5+0,5 . \mathrm{P}_{\mathrm{MOD}} \sin 2 \pi \mathrm{F}_{\mathrm{BF}}$.

Pour le $2^{\text {ème }}$ bras $\left(\mathrm{T}_{2 \mathrm{~A}}\right.$ et $\left.\mathrm{T}_{2 \mathrm{~B}}\right)$ le rapport cyclique est $\alpha_{2}=0,5+0,5 . \mathrm{P}_{\mathrm{MOD}} \sin \left(2 \pi \mathrm{F}_{\mathrm{BF}}+2 \pi / 3\right)$. 
Pour le $3^{\text {ème }}$ bras $\left(\mathrm{T}_{3 \mathrm{~A}}\right.$ et $\left.\mathrm{T}_{3 \mathrm{~B}}\right)$ le rapport cyclique est $\alpha_{3}=0,5+0,5 . \mathrm{P}_{\mathrm{MOD}} \sin \left(2 \pi \mathrm{F}_{\mathrm{BF}}+4 \pi / 3\right)$.

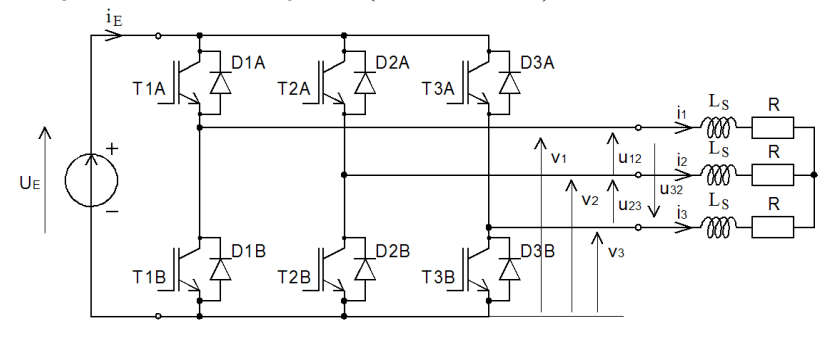

Fig 18: Onduleur triphasé.

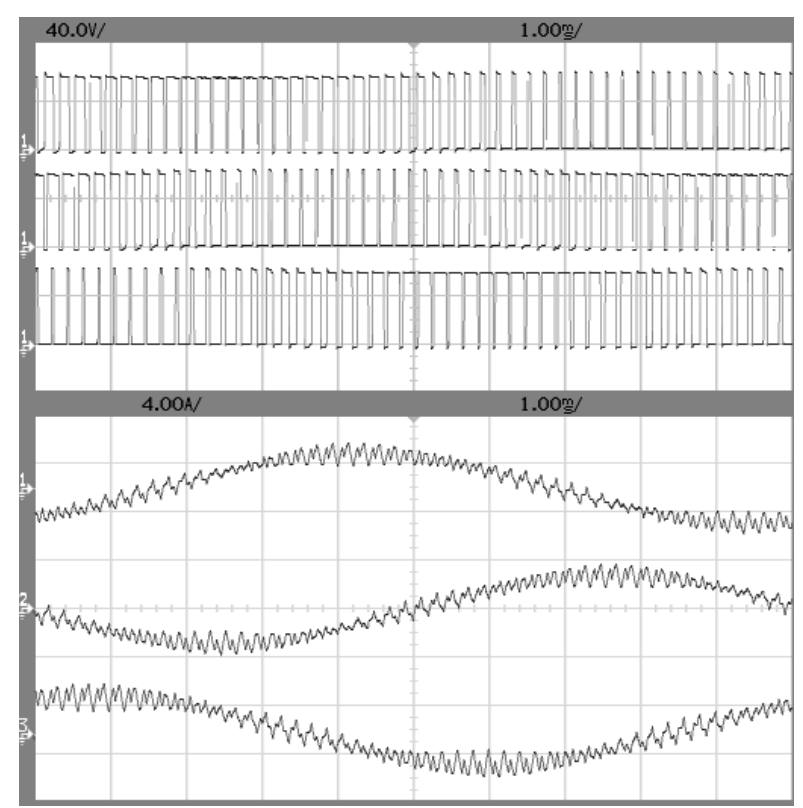

Fig 19 : Onduleur triphasé. Haut : les 3 tensions de sortie $v_{1} . v_{2}$ et $v_{3}$. Bas : courants de sortie $i_{1}, i_{2}$ et $i_{3}$.

\subsection{Onduleur triphasé avec $\mathrm{U} /=$ Cte}

Ce mode de commande permet l'alimentation de moteurs asynchrones triphasés. Le fonctionnement est équivalent à l'onduleur triphasé simple mais l'amplitude de modulation n'est plus réglable indépendamment, elle est lié à la fréquence de modulation selon une loi de type $\mathrm{U} / \mathrm{f}=$ constante.

\subsection{Onduleur pour chauffage par induction}

Dans ce mode un seul bras est commandé avec un rapport cyclique fixe $\alpha=0,5$ et une fréquence de découpage réglable entre $20 \mathrm{kHz}$ et $50 \mathrm{kHz}$ par pas de $100 \mathrm{~Hz}$. Remarque : en réalité les 3 bras sont commandé avec le même signal, ainsi une mise en parallèle des 3 bras permet d'augmenter la valeur maximale du courant de sortie à environ $50 \mathrm{~A}$ crête. Il est également possible de commander un pont monophasé avec le même rapport cyclique $\alpha=0,5$. Ceci permet de doubler la tension appliquée à la charge mais avec une valeur maximale de courant plus faible. La figure 20 montre un exemple de fonctionnement sur une plaque à induction avec une puissance de sortie de $2,1 \mathrm{~kW} \quad\left(\mathrm{U}_{\mathrm{DC}}=270 \mathrm{~V}\right.$, $\left.\mathrm{F}_{\text {déc }}=31,7 \mathrm{kHz}, \mathrm{I}_{\text {eff }}=18,3 \mathrm{~A}\right)$.

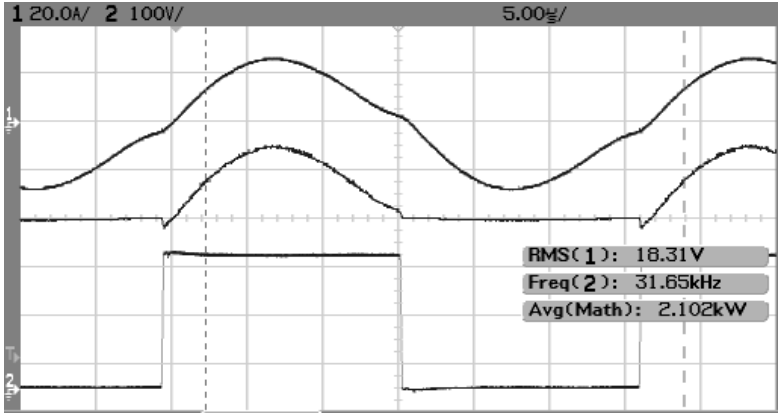

Fig 20: Chauffage par induction HF. De haut en bas: courant inducteur (20A/div), puissance instantanée $(5 \mathrm{~kW} / \mathrm{div})$, et tension onduleur (100V/div).

\subsection{Onduleur triphasé connecté au réseau}

Dans le cadre de projets de master M1 nous avons également fait travailler des étudiants sur un onduleur triphasé connecté au réseau en utilisant cet l'ensemble hacheur-onduleur légèrement modifié. Il s'agit d'un dispositif simple et pédagogique fonctionnant en boucle ouverte. Tous les réglages sont assurés par l'utilisateur en fonction des indications des instruments. Pour ce fonctionnement la fréquence de modulation est verrouillée à $50 \mathrm{~Hz}$. Le générateur $\mathrm{BF}$ interne est synchronisé sur la tension réseau par un détecteur de passage par zéro. L'onduleur est connecté au réseau avec un inductance triphasée présentant à $50 \mathrm{~Hz}$ une impédance d'environ $20 \%$ du rapport $\mathrm{V} / \mathrm{I}$, tension réseau sur amplitude du courant injecté. Le réglage de l'amplitude de la tension de sortie agit essentiellement sur le courant réactif injecté (ou soutiré) au réseau. Le réglage du déphasage de la tension onduleur par rapport à la tension réseau agit essentiellement sur le courant actif injecté au réseau.

\section{CONCLUSION}

L'ensemble expérimental présenté ici s'est révélé tout à fait adapté à nos objectifs pédagogiques. Ces TP sont très bien perçus par les étudiants. Le circuit de commande performant basé autour d'un FPGA contribue à véhiculer une image moderne et permet une liaison avec les enseignements d'électronique.

Nous n'avons eu à déplorer que peu de défaillances. Elles étaient généralement dues à une utilisation plus que incorrecte de la part des étudiants! La maintenance est facile et relativement peu coûteuse avec une localisation des pannes assez aisée.

\section{Bibliographie}

1. Site web ALTERA : http://www.altera.com.

2. F. Forest, J.-J. Huselstein, F. Richardeau, C. Turpin, La méthode d'opposition: une vieille dame rajeunie par les besoins de caractérisation et d'étude des convertisseurs statiques, La Revue 3EI, No31, pp 33-40, décembre 2002. 